\title{
Scaling and Universality of Critical Fluctuations in Granular Gases
}

\author{
J. Javier Brey, ${ }^{*}$ M. I. García de Soria, P. Maynar, and M. J. Ruiz-Montero \\ Física Teórica, Universidad de Sevilla, Apartado de Correos 1065, E-41080 Sevilla, Spain
}

(Received 23 November 2004; published 10 March 2005)

\begin{abstract}
The total energy fluctuations of a low-density granular gas in the homogeneous cooling state near the threshold of the clustering instability are studied by means of molecular dynamics simulations. The relative dispersion of the fluctuations is shown to exhibit a power-law divergent behavior. Moreover, the probability distribution of the fluctuations presents data collapse as the system approaches the instability, for different values of the inelasticity. The function describing the collapse turns out to be the symmetric of the one found in several molecular equilibrium and nonequilibrium systems.
\end{abstract}

DOI: 10.1103/PhysRevLett.94.098001

PACS numbers: 45.70.-n, 05.20.Dd, 51.10.+y

A granular system is an assembly of macroscopic particles dissipating their energy through inelastic collisions. In the rapid granular flow regime, the grains move freely and independently between collisions and the system is often referred to as a granular gas. In this regime, the behavior of a granular system resembles that of a molecular fluid, although with many significant differences [1]. These discrepancies have been extensively illustrated by using theoretical, experimental, and also particle simulation methods [2]. In this Letter, evidence is reported of a rather surprising analogy between granular gases and molecular systems. It is shown that the statistical behavior of a freely evolving granular system near the clustering instability has strong similarities with that of several molecular model systems, including equilibrium systems in the critical region. More precisely, the second moment of the relative fluctuations of the total energy diverges with a given critical exponent. Besides, and even more remarkably, the energy fluctuations obey a scaling law that, when properly expressed, is the same as the one found in some correlated equilibrium and nonequilibrium molecular systems [3-5].

A widely used simple model for granular gases is a system of smooth inelastic hard spheres $(d=3)$ or disks $(d=2)$ of mass $m$ and diameter $\sigma$. The inelasticity of collisions is characterized by a constant coefficient of normal restitution $\alpha$. As a consequence of the energy dissipation in collisions, isolated granular gases do not exhibit any homogeneous time-independent state. The simplest state is the so-called homogeneous cooling state (HCS) [6] with vanishing flow field and a monotonically decreasing temperature $T_{\mathrm{HCS}}(t)$, obeying the equation $\partial_{t} T_{\mathrm{HCS}}(t)=-\zeta_{\mathrm{HCS}}\left(T_{\mathrm{HCS}}\right) T_{\mathrm{HCS}}(t)$, where $\zeta_{\mathrm{HCS}}\left(T_{\mathrm{HCS}}\right) \propto$ $T_{\mathrm{HCS}}^{1 / 2}$ is the cooling rate. At a microscopic level, it has been postulated that the time dependence of the ensemble describing this state occurs only through the scaling of the velocities with the thermal velocity, that is proportional to $T_{\mathrm{HCS}}^{1 / 2}(t)$, and the corresponding normalization [7]. The HCS is unstable against long wavelength spatial perturbations [8], leading to the spontaneous formation of velocity vortices and density clusters. This clustering instability is accurately predicted by a linear stability analysis of the hydrodynamic equations, which shows that it is driven by the transversal shear mode [8,9]. A critical length $L_{c}$ is identified, so that the system becomes unstable when its linear size $L$ exceeds $L_{c}$. Its value has been determined for a low-density granular gas described by the (inelastic) Boltzmann equation, and it is given by [10]

$$
L_{c}=\frac{(2+d) \Gamma(d / 2)}{2 \pi^{(d-3) / 2} n \sigma^{(d-1)}}\left(\frac{\eta^{*}}{2 \zeta^{*}}\right)^{1 / 2},
$$

where $n$ is the number of particles density, $\zeta^{*}(\alpha)=$ $\eta_{0} \zeta_{\mathrm{HCS}} / n k_{B} T_{\mathrm{HCS}}$, and $\eta^{*}(\alpha)=\eta\left(T_{\mathrm{HCS}}\right) / \eta_{0}\left(T_{\mathrm{HCS}}\right)$, with $k_{B}$ being the Boltzmann constant, $\eta$ the shear viscosity of the granular gas, and $\eta_{0}$ its elastic limit. The explicit expressions of $\zeta^{*}$ and $\eta^{*}$ are given in [10]. In the elastic limit $\alpha=1, \zeta^{*}$ vanishes, and $\eta^{*}=1$. The accuracy of this prediction has been verified by direct Monte Carlo simulation of the Boltzmann equation [11].

Another important consequence of the inelasticity of collisions, largely unexplored, is the presence of an intrinsic noise in the macroscopic description of the system. Recently [12], it has been shown that the total energy of a dilute granular gas in the HCS exhibits fluctuations and time-correlation properties which are caused by the energy dissipation in collisions and, at a mesoscopic level, by the presence of velocity correlations. Although the correlations increase as the inelasticity increases, they remain relatively small over all the range of values of $\alpha$ studied $(\alpha \geq 0.6)$. Here it is shown that the noise is amplified, and, in fact, it diverges at the threshold of the clustering instability.

We have performed molecular dynamics (MD) simulations of a freely evolving system of $N$ inelastic hard disks $(d=2)$, using the steady-state method, which is based on an exact mapping of the HCS onto a steady state. This property follows from a change in the time scale, as discussed in detail in [13]. The steady representation of the HCS removes the difficulties associated with the rapid cooling of the fluid leading to numerical inaccuracies 
very soon. With this method, the trajectories can be followed for an arbitrary time. The system considered has been a square box of size $L$ with periodic boundary conditions. The density in all the results reported in the following is $n=0.02 \sigma^{-2}$, so the values of $L$ and $N$ were consistently changed in the different simulations. The above value of the density has been found to be low enough as to guarantee that the average behavior of the system is accurately described by the Boltzmann equation, even in the unstable region of the granular gas [14]. Two different values of the restitution coefficient have been considered, $\alpha=0.8$ and $\alpha=0.9$, for which Eq. (1) gives $L_{c} \simeq 304 \sigma$ and $L_{c} \simeq 413 \sigma$, respectively. We have studied the behavior of the total properties of the system when the critical size is approached from below by increasing the value of $L$ at constant $\alpha$ (and $n$ ). In all the simulations, we have checked that the system was actually in the HCS by monitoring the local velocity and density fluctuations.

The first quantity we have investigated is the reduced cooling rate $\zeta^{*}$ as a function of the linear size of the system, at constant $\alpha$. The simulations show that it monotonically decreases very slowly as $L$ approaches its critical value. The decay is imperceptible on the scale used in Fig. 1, where $\ln \zeta^{*-2}$ is plotted as a function of $\widetilde{\delta L} \equiv\left(L_{c}-L\right) / L_{c}$ for $\alpha=0.9$. Here and in the following, the theoretical prediction for $L_{c}$ given by Eq. (1) has been used. To make the above dependence on $L$ explicit, we have also plotted $\ln \left[\zeta^{*-2}(L)-\zeta^{*-2}(0)\right]$, where $\zeta^{*}(0)$ is the constant asymptotic value of the reduced cooling rate far below the transition point, also obtained from the simulations. The results indicate a behavior of the form $\zeta^{*-2}(L) \sim$

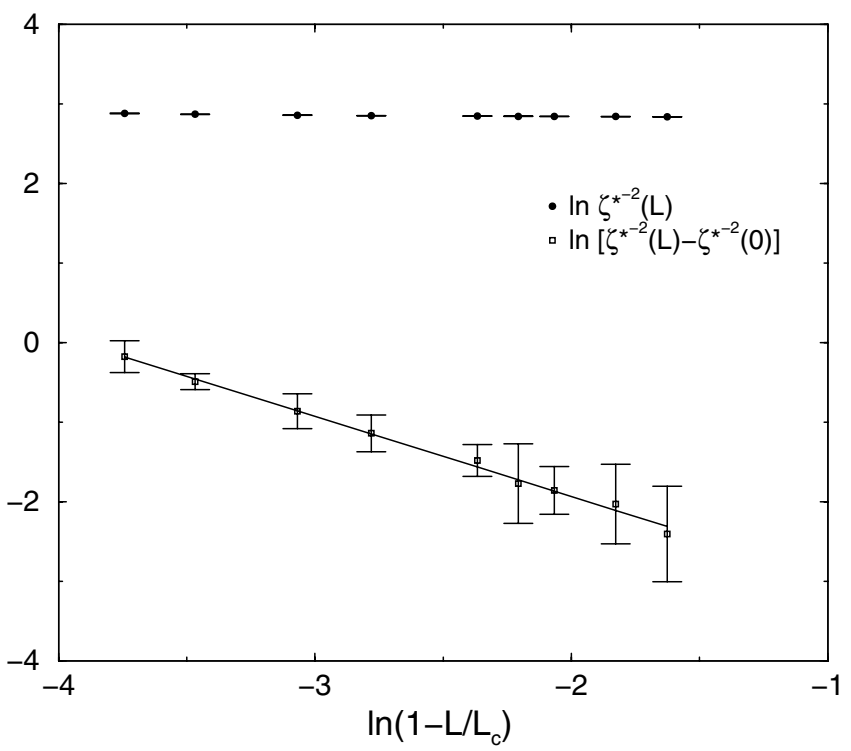

FIG. 1. Dependence of the scaled cooling rate $\zeta^{*}(L)$ on the "distance" to the instability point $\widetilde{\delta L} \equiv 1-L / L_{c}$ for a system of inelastic hard disks with $\alpha=0.9$. The solid line is a fit to the power-law behavior discussed in the text.
$\zeta^{*-2}(0)+A_{\zeta} \widetilde{\delta L}^{-1}$, with $A_{\zeta} \simeq 0.02$. Similar results were found for $\alpha=0.8$. If this behavior persists until the instability point, it would imply that the cooling rate tends to vanish as $\left(\widetilde{\delta L} / A_{\zeta}\right)^{1 / 2}$. Nevertheless, since in the observed region $\zeta^{*}(L)$ is dominated by its asymptotic part $\zeta^{*}(0)$, it is difficult to make any definite statement on this point.

Next, let us consider the second moment of the fluctuations of the total energy $E$,

$$
\sigma_{E}^{2} \equiv \frac{\left\langle E^{2}(t)\right\rangle-\langle E(t)\rangle^{2}}{\langle E(t)\rangle^{2}}
$$

where the angular brackets denote average over the ensemble generated by a number of trajectories [12]. The above quantity does not depend on time in the HCS, due to the scaling property of its distribution function. The results, again for $\alpha=0.9$, are given in Fig. 2. For $L \ll L_{c}$, $N \sigma_{E}^{2}(L)$ is practically constant, independent of $L$, indicating that $\sigma_{E} \propto N^{-1 / 2}$, as expected. In fact, its value in this region, which we denote by $N \sigma_{E}^{2}(0)$, is accurately predicted by the result derived in Ref. [12] by using kinetic theory methods. Although it is a function of $\alpha$, increasing as $\alpha$ decreases, it remains rather small, at least for not too inelastic systems. On the other hand, when $L$ approaches its critical value, the energy fluctuations grow very fast. This is clearly seen by studying the quantity $N\left[\sigma_{E}^{2}(L)-\right.$ $\left.\sigma_{E}^{2}(0)\right]$, which is also plotted in the same figure. It follows that close enough to the instability point, the second moment of the total energy fluctuations is accurately described by the critical law

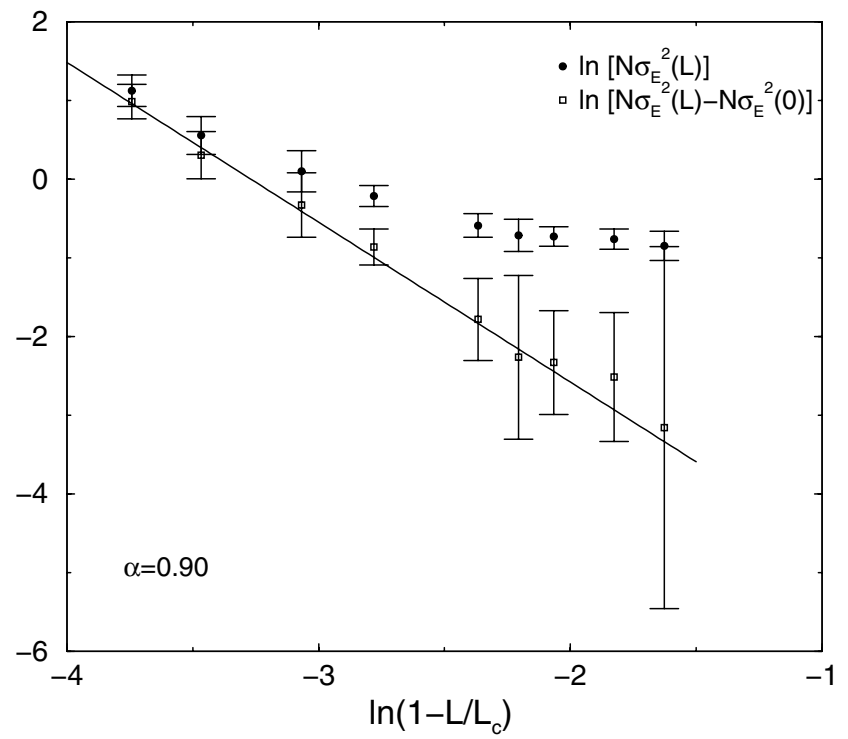

FIG. 2. Scaled second moment of the total energy fluctuations $\sigma_{E}^{2}$ as a function of the distance $\widetilde{\delta L}$ to the instability point for $\alpha=0.9$. Note that the second moment has been multiplied by the number of particles $N$. The solid line is the fit to the power law defined in the text. 


$$
\sigma_{E}(L) \sim A_{E}(\widetilde{\delta L})^{-1}
$$

where $A_{E} \simeq 6 \times 10^{-4}$ is a critical amplitude. Upon writing the above equation, we have used that near the instability point we can approximate $N \sim N_{c}=n L_{c}^{2}$. The same behavior is found for $\alpha=0.8$, but with the amplitude $A_{E} \simeq$ $1.5 \times 10^{-3}$. Therefore, the simulation results indicate a divergent critical behavior of the fluctuations, with a critical exponent -1 and an amplitude that depends on the value of the coefficient of restitution $\alpha$. Of course, the validity of the above relies on the assumption that the observed behavior remains the same until the system is asymptotically close to the instability. It must be pointed out that close enough to $L_{c}$, the above divergence might be arrested by nonlinearities as it happens in numerous other systems. In fact, preliminary simulation results indicate that this may be the case, although no definitive conclusion can be presently established.

To analyze in more detail the structure of the energy fluctuations, we make the unjustified assumption that the probability distribution function (PDF) $P_{L}(\widetilde{\delta E})$ of the relative energy fluctuations $\widetilde{\delta E} \equiv[E(t)-\langle E(t)\rangle] /\langle E(t)\rangle$ in the vicinity of the clustering instability verifies the scaling relation

$$
P_{L}(\widetilde{\delta E})=\frac{1}{\sigma_{E}} \tilde{f}\left(\frac{\widetilde{\delta E}}{\sigma_{E}}\right)
$$

where $\tilde{f}$ is a scaling function. It is moreover assumed that all the dependence of $\tilde{f}$ on $\alpha$ and $L$ occurs through $\sigma_{E}$. Consider then the function

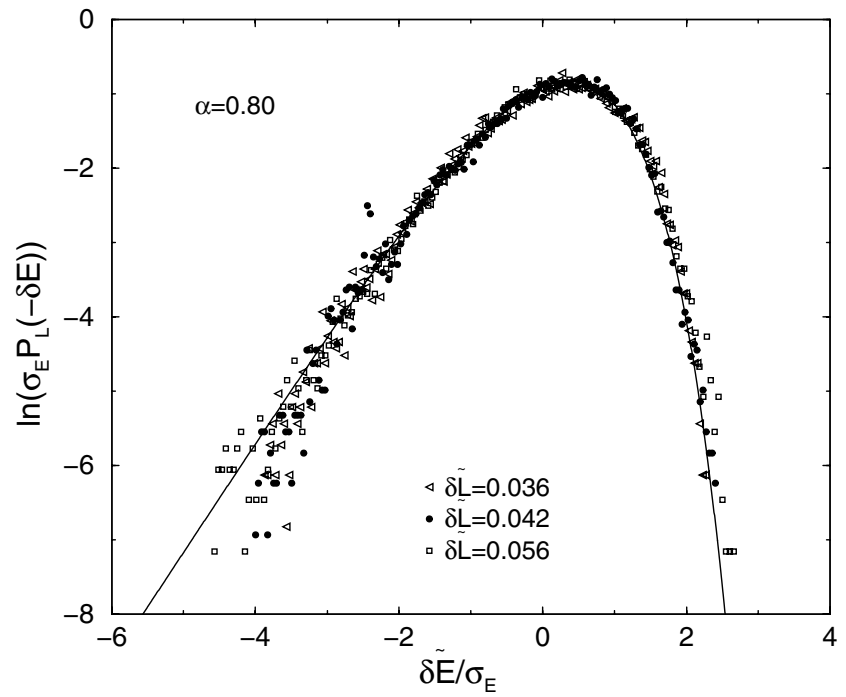

FIG. 3. Probability density function of the relative total energy fluctuations $\sigma_{E} P_{L}(-\widetilde{\delta E})$ for a system of inelastic hard disks with $\alpha=0.8$. The symbols are from MD simulations and the solid line is Eq. (6).

$$
\sigma_{E} P_{L}(\widetilde{\delta E})=\tilde{f}\left(\frac{\widetilde{\delta E}}{\sigma_{E}}\right)
$$

All the $L$ and $\alpha$ dependence has been eliminated on the right-hand side of the above expression when considered as a function of $\widetilde{\delta E} / \sigma_{E}$. Thus as long as the assumed scaling holds near the clustering instability, the data for systems with different sizes should tend to collapse onto each other as $L$ approaches $L_{c}$. Moreover, the collapse must occur on a function that does not depend on $\alpha$. To check this strong prediction, we have computed the PDF of the fluctuations in the MD simulations. For each trajectory of a system, the range of values of $\widetilde{\delta E}$ has been partitioned into nonoverlapping bins and the frequency distribution has been built up using all the trajectories corresponding to the same values of $L$ and $\alpha$. The resulting normalized distributions are plotted in Figs. 3 and 4, for $\alpha=0.8$ and $\alpha=0.9$, respectively. In each case, results for different system sizes are given. Notice that, for reasons that are explained later, what has been actually plotted is $\sigma_{E} P_{L}(-\widetilde{\delta E})$. The data are seen to fall quite closely on top of each other, as implied by the scaling law (5), over more than 3 orders of magnitude, specially when attention is restricted to the results for the two largest systems. Moreover, the fluctuations are highly non-Gaussian and asymmetric around the mean value. This is a manifestation of the presence of a correlation length of the order of the size of the system, so that it cannot be divided into statistical independent mesoscopic regions and, therefore, there is no reason to expect the fluctuations of global quantities to be Gaussian.

The shape of PDF's in Figs. 3 and 4 looks quite similar to the functional form found in other equilibrium and nonequilibrium systems $[3,4]$. To make this statement more precise, we have considered the approximated form

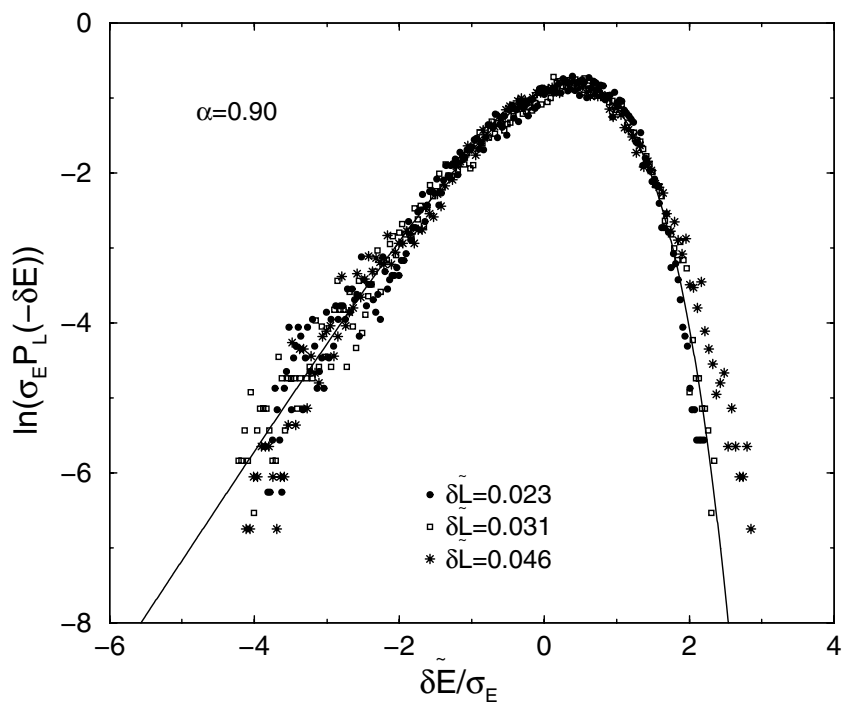

FIG. 4. The same as in Fig. 3, but for $\alpha=0.9$. 
of the PDF for the fluctuations of the total magnetization in the two-dimensional $X Y$ model in the harmonic approximation, for temperatures well below the KosterlitzThouless transition [4],

$$
\Pi(y)=K\left(e^{x-e^{x}}\right)^{a}, \quad x=b(y-s), \quad a=\pi / 2 .
$$

The high $y$ region of this function is approximately Gaussian, while it presents an exponential tail for large negative values. A quite large number of nonequilibrium systems exhibiting self-organized criticality has been found to show data collapse with a PDF very similar to $\Pi(y)$ [4]. Quite remarkably, the same kind of behavior had been previously observed in experiments with confined turbulent flows [15]. In the present context, in principle, it should be $\Pi(y)=\sigma_{E} P_{L}(y)$ with $y=\widetilde{\delta E}$, but in order to get a good agrement with the MD results, we have to change $\widetilde{\delta E}$ by $-\widetilde{\delta E}$ in the identification of $y$. The values of the three parameters in Eq. (6) follow from the normalization, the zero mean, and unit variance conditions, with the results $K=2.14, \quad b=0.938$, and $s=0.374$. Therefore, Eq. (6) has no fitting parameters. The function $\Pi(y)$ has been also plotted in Figs. 3 and 4, and a surprisingly good agreement with the simulation data is found. The change in the sign of the fluctuations, i.e., the fact that the energy fluctuations in a granular gas are described by a function that is the symmetric with respect to the origin of that for ordinary systems, may be due to the dissipative character of granular systems. In the HCS the gas is continuously dissipating energy, while in molecular systems energy must be continuously supplied in order to keep them in a nonequilibrium steady state. Let us mention that large fluctuations around the threshold of a symmetry breaking instability have also been observed recently in granular systems driven by a thermal sidewall at zero gravity [16]. To our knowledge, whether these fluctuations can be scaled in a similar way to the one described here has not been investigated yet.

The natural question prompted by the results reported in this work is whether the agreement of the fluctuation spectra of such a variety of systems is telling us something about the intrinsic behavior of a quite general class of systems. If this is the case, how this class can be characterized a priori, and the divergent behavior (3) and the specific scaling form (6), or another one very close to it, can be derived on the ground of general arguments are open questions. Specifically in the context of granular systems, how can the above results be obtained starting from a microscopic description of the system? Are they captured by a fluctuating hydrodynamics theory? Is there an underlying hyperscaling in the sense discussed in [5]?
Finally, it must be stressed that we have restricted ourselves here to two-dimensional low-density granular gases. Although it seems that the same kind of results can be expected at higher densities and also for three-dimensional systems (perhaps with a different critical exponent), this is something to be verified. These points are presently under study and some results will be reported elsewhere.

This research was supported by the Ministerio de Ciencia y Tecnología (Spain) through Grant No. BFM2002-00307 (partially financed by FEDER funds).

*Electronic address: brey@us.es

[1] See, for instance, H.M. Jaeger, S. R. Nagel, and R. P. Behringer, Rev. Mod. Phys. 68, 1259 (1996).

[2] For a review and references, see I. Goldhirsch, Annu. Rev. Fluid Mech. 35, 267 (2003).

[3] S. T. Bramwell, P.C.W. Holdsworth, and J.-F. Pinton, Nature (London) 396, 552 (1998).

[4] S. T. Bramwell, K. Christensen, J.-Y. Fortin, P.C.W. Holdsworth, H. J. Jensen, S. Lise, J. M. López, M. Nicodemi, J.-F. Pinton, and M. Sellitto, Phys. Rev. Lett. 84, 3744 (2000).

[5] V. Aji and N. Goldenfeld, Phys. Rev. Lett. 86, 1007 (2001).

[6] P. K. Haff, J. Fluid Mech. 134, 401 (1983).

[7] A. Goldshtein and M. Shapiro, J. Fluid Mech. 282, 75 (1995); J. J. Brey, J. W. Dufty, and A. Santos, J. Stat. Phys. 87, 1051 (1997).

[8] I. Goldhirsch and G. Zanetti, Phys. Rev. Lett. 70, 1619 (1993); S. McNamara and W. R. Young, Phys. Rev. E 50, R28 (1994).

[9] J. J. Brey, M. J. Ruiz-Montero, and D. Cubero, Phys. Rev. E 60, 3150 (1999).

[10] J. J. Brey, J. W. Dufty, C. S. Kim, and A. Santos, Phys. Rev. E 58, 4638 (1998); J. J. Brey and D. Cubero, in Granular Gases, edited by T. Pöschel and S. Luding, Lecture Notes in Physics (Springer, Berlin, 2000).

[11] J. J. Brey, M. J. Ruiz-Montero, and F. Moreno, Phys. Fluids 10, 2976 (1998).

[12] J. J. Brey, M. I. García de Soria, P. Maynar, and M. J. RuizMontero, Phys. Rev. E 70, 011302 (2004).

[13] J. F. Lutsko, Phys. Rev. E, 63, 061211 (2001); J. J. Brey, M. J. Ruiz-Montero, and F. Moreno, Phys. Rev. E, 69, 051303 (2004).

[14] J. J. Brey and M.J. Ruiz-Montero, Phys. Rev. E 69, 011305 (2004).

[15] J.-F. Pinton, P. C. W. Holdsworth, and R. Labbé, Phys. Rev. E 60, R2452 (1999).

[16] B. Meerson, T. Pöschel, P. V. Sasorov, and T. Schwager, Phys. Rev. E 69, 021302 (2004). 